UPRF 96-484

IFUM-536-FT

August 1996

\title{
Gauge invariant action at the ultraviolet cutoff ${ }^{*}$
}

\author{
M. Bonini \\ Dipartimento di Fisica, Università di Parma and \\ INFN, Gruppo Collegato di Parma, Italy \\ and \\ G. Marchesini \\ Dipartimento di Fisica, Università di Milano and \\ INFN, Sezione di Milano
}

\begin{abstract}
We show that it is possible to formulate a gauge theory starting from a local action at the ultraviolet (UV) momentum cutoff which is BRS invariant. One has to require that fields in the UV action and the fields in the effective action are not the same but related by a local field transformation. The few relevant parameters involved in this transformation (six for the $S U(2)$ gauge theory), are perturbatively fixed by the gauge symmetry.
\end{abstract}

\footnotetext{
*Research supported in part by MURST, Italy and by EC Programme "Human Capital and Mobility",
} contract CHRX-CT93-0357 (DG 12 COMA). 
Consider the UV (or bare) action $S_{\Lambda_{0}}[\phi, \gamma]$ of a Yang-Mills theory with $\Lambda_{0}$ the UV cutoff ( $\phi$ represents the gauge, ghost and antighost fields while $\gamma$ are the BRS sources [1]). Within the exact Wilson renormalization group ( $R G$ ) formulation [2]-[5] this UV action can be viewed as the result of taking an elementary underlying theory and integrating all degrees of freedom with frequencies larger than the UV cutoff. For $\Lambda_{0}$ much larger than any physical momentum, $S_{\Lambda_{0}}[\phi, \gamma]$ can be taken local and given by few "relevant" parameters] which are the only trace of the underlying theory.

By using the exact Wilson RG it has been shown [5, 6] that it is possible to compute perturbatively the relevant parameters of $S_{\Lambda_{0}}[\phi, \gamma]$ in such a way that the effective action $\Gamma[\phi, \gamma]$, obtained by integrating over the fields with frequencies smaller than $\Lambda_{0}$, satisfies the Slavnov-Taylor (ST) identities in the limit $\Lambda_{0} \rightarrow \infty$. This is obtained by properly fixing the relevant parameters of $\Gamma[\phi, \gamma]$ and then computing the large $\Lambda_{0}$ dependence of the relevant parameters of the UV action.

Although it is beautiful to see that by fixing the few parameters in $S_{\Lambda_{0}}[\phi, \gamma]$ one obtains the functional identity for $\Gamma[\phi, \gamma]$ which is the consequence of the gauge symmetry, it is however ugly the fact that the UV action itself does not manifest the gauge symmetry in a simple way. For example in $S_{\Lambda_{0}}[\phi, \gamma]$ one needs to include an appropriate mass term for the gauge field. The UV action may be considered as the closest functional to the underlying theory, but it does not have any explicit symmetry.

In this paper we show that it is actually possible to formulate the theory in such a way that both the UV action is BRS invariant and the effective action satisfies the ST identities. To obtain this we try to adapt to the continuous case the formulation of lattice gauge theory (LGT) [7] in which the UV action and the path integral measure are explicitly gauge invariant. They are expressed in terms of link variables given by exponential of the gauge fields on the lattice. As we shall see the key point we exploit and adapt to the continuous case is the non linear relation between the link and field variables.

\section{UV action and BRS symmetry}

We start by introducing the UV action in the continuum and, as an example, we consider the $S U(2)$ Yang-Mills theory. Inspired by LGT we are ready to distinguish between the fields $\phi=\left(A_{\mu}, c, \bar{c}\right)$ which enter into the physical observables, i.e. in the effective action $\Gamma[\phi, \gamma]$, and the UV fields $\Phi=\left(\mathcal{A}_{\mu}, c, \bar{c}\right)$, which enter into the UV action?, the analogous of the link variables in LGT. At the UV scale $\Lambda_{0}$ we assume that the UV action is given by the BRS classical action, e.g. in the Feynman gauge,

$$
S_{\mathrm{BRS}}\left[\Phi, \gamma ; g_{\Lambda_{0}}\right]=\int d^{4} x\left\{-\frac{1}{4} F_{\mu \nu}^{2}-\frac{1}{2}\left(\partial_{\mu} \mathcal{A}_{\mu}\right)^{2}+W_{\mu} \cdot D_{\mu} c-\frac{1}{2} v \cdot c \wedge c\right\}
$$

with $F_{\mu \nu}=\partial_{\mu} \mathcal{A}_{\nu}-\partial_{\nu} \mathcal{A}_{\mu}+g_{\Lambda_{0}} \mathcal{A}_{\mu} \wedge \mathcal{A}_{\nu}, \quad D_{\mu} c=\partial_{\mu} c+g_{\Lambda_{0}} \mathcal{A}_{\mu} \wedge c, W_{\mu}=\partial_{\mu} \bar{c}+u_{\mu} / g_{\Lambda_{0}}$, and the UV coupling $g_{\Lambda_{0}}$. We have introduced the usual scalar and external $S U(2)$ products and the sources $\gamma=\left(u_{\mu}, v\right)$ for the BRS variations of $\mathcal{A}_{\mu}$, and $c$ respectively.

\footnotetext{
${ }^{2}$ In a functional, the coefficients of local field monomials with non-negative dimension will be called relevant parameters [2].

${ }^{3}$ It is not necessary to introduce different fields for the ghost and the antighost. See later.
} 
In the path integral over the field $\Phi(x)$ we have to consider only frequencies smaller than $\Lambda_{0}$. To cutoff larger frequencies one could for instance add [3]- [6] to the UV action the following contribution (in terms of the Fourier transform of the fields)

$$
S_{\text {cutoff }}[\Phi] \equiv-\int \frac{d^{4} p}{(2 \pi)^{4}} p^{2}\left(K_{\Lambda_{0}}^{-1}(p)-1\right)\left\{\frac{1}{2} \mathcal{A}_{\mu}^{\mathrm{R}}(-p) \cdot \mathcal{A}_{\mu}^{\mathrm{R}}(p)-c^{\mathrm{R}}(-p) \cdot c^{\mathrm{R}}(p)\right\},
$$

in which we have introduced the cutoff function $K_{\Lambda_{0}}(p)=1$ for $p^{2}<\Lambda_{0}^{2}$ and rapidly vanishing for larger frequencies. The contribution $S_{\text {cutoff }}[\Phi]$ vanishes if the fields are in the low momentum region $\left(p^{2}<\Lambda_{0}^{2}\right)$ while diverges if the fields have large frequencies $\left(p^{2}>\Lambda_{0}^{2}\right)$. As a consequence, in the path integral the large frequency fields are suppressed. $S_{\text {cutoff }}[\Phi]$ is not BRS invariant, however its variation always involves large frequency fields which are suppressed in the path integral.

For conveniency (see [6]) we have introduced in (2) the "renormalized" fields $\Phi^{\mathrm{R}}$ related to the UV fields $\Phi$ by multiplicative constants to be discussed later.

\section{Ultraviolet and effective fields}

We have now to establish the relation between the "UV field" $\mathcal{A}_{\mu}(x)$ entering into the UV action, and the "effective field" $A_{\mu}(x)$ entering into the effective action $\Gamma[\phi, \gamma]$. In general one should assume that the two fields are the same within a distance $1 / \Lambda_{0}$. The UV field $\mathcal{A}_{\mu}(x)$ is then given by a functional of the effective field $A_{\mu}(y)$ with $y$ confined in a region $|x-y|<1 / \Lambda_{0}$. By expanding around $x$ one finds

$$
\mathcal{A}_{\mu}(x)=A_{\mu}(x)\left\{1-\Lambda_{0}^{-2} \Delta_{2}(A(x))-\Lambda_{0}^{-4} \Delta_{4}(A(x))+\cdots\right\},
$$

where the dots denote terms with higher powers of $1 / \Lambda_{0}$. The functions $\Delta_{2}(A)$ and $\Delta_{4}(A)$ of dimension two and four respectively are polynomials in the fields $A_{\nu}$ which are scalars under the Lorentz and $S U(2)$ transformations. This requirement fixes the polynomials in terms of six parameters

$$
\begin{aligned}
\Delta_{2}(A)= & \frac{1}{2} c_{5} A_{\nu} \cdot A_{\nu} \\
\Delta_{4}(A)= & \frac{1}{2} c_{0} A_{\nu} \cdot \partial^{2} A_{\nu}-\frac{1}{2} c_{1}\left(\partial_{\nu} A_{\nu}\right)^{2}+c_{2}\left(\partial_{\nu} A_{\rho}\right) \cdot A_{\rho} \wedge A_{\nu} \\
& +\frac{1}{4} c_{3}\left(A_{\rho} \wedge A_{\nu}\right) \cdot\left(A_{\nu} \wedge A_{\rho}\right)+\frac{1}{4} c_{4}\left(A_{\nu} \cdot A_{\nu}\right)^{2} .
\end{aligned}
$$

Formally, in the limit $\Lambda_{0} \rightarrow \infty$ the UV and effective fields coincide. However this is not the case and the limit is not trivial, as we shall see.

In LGT, the UV field $\mathcal{A}_{\mu}(x)$ corresponds to the link variable $U_{\mu}(x)$ and the field transformation (3i) corresponds to the exponential mapping $U_{\mu}(x)=\exp \left\{g_{\text {lat }} a A_{\mu}(x)\right\}$ with $g_{\text {lat }}$ the lattice gauge coupling and $a$ the lattice size. This transformation has the advantage that it induces a group structure, thus one can easily prove that the lattice effective action satisfies ST identities [8]. However it cannot be applied in a simple way to the continuous case since in the exponential mapping Lorentz covariance is lost. In the continuous case the transformation (3) preserves Lorentz covariance and the expansion parameters $c_{i}$ will be fixed by requiring ST identities for the effective action $\Gamma[\phi, \gamma]$. 
Consider the BRS transformation of the elementary field $\Phi$ which leaves invariant the UV action (11) and the measure $D \Phi$. For the gauge field one has

$$
\delta_{\mathrm{BRS}} \mathcal{A}_{\mu}(x)=\eta\left\{\partial_{\mu} c+g_{\Lambda_{0}} \mathcal{A}_{\mu}(x) \wedge c(x)\right\}
$$

with $\eta$ the Grassmann parameter. For the effective field this transformation becomes

$$
\delta_{\mathrm{BRS}} A_{\mu}(x)=\left\{\delta_{\mathrm{BRS}}^{(0)}+\Lambda_{0}^{-2} \delta_{\mathrm{BRS}}^{(2)}+\Lambda_{0}^{-4} \delta_{\mathrm{BRS}}^{(4)}+\cdots\right\} A_{\mu}(x),
$$

where

$$
\begin{aligned}
& \delta_{\mathrm{BRS}}^{(0)} A_{\mu}(x)=\eta\left\{\partial_{\mu} c+g_{\Lambda_{0}} A_{\mu}(x) \wedge c(x)\right\} \\
& \delta_{\mathrm{BRS}}^{(2)} A_{\mu}(x)=\eta c_{5}\left\{\partial_{\mu} c(x)\left(\frac{1}{2} A_{\nu}(x) \cdot A_{\nu}(x)\right)+A_{\mu}(x)\left(\partial_{\nu} c(x) \cdot A_{\nu}(x)\right)\right\},
\end{aligned}
$$

and a more complex expression for $\delta_{\mathrm{BRS}}^{(4)} A_{\mu}(x)$. Formally the BRS transformation of $A_{\mu}(x)$ and $\mathcal{A}_{\mu}(x)$ are the same in the limit $\Lambda_{0} \rightarrow \infty$. However, due to the non linearity of (3), the Jacobian of the transformation is not trivial even for $\Lambda_{0} \rightarrow \infty$. To compute the Jacobian consider

$$
\frac{\delta \mathcal{A}_{\mu}^{a}(x)}{\delta A_{\nu}^{b}(y)}=\delta^{4}(x-y)\left\{\delta_{a b} \delta_{\mu \nu}-\Lambda_{0}^{-2} c_{5}\left[\delta_{a b} \delta_{\mu \nu} \frac{1}{2} A_{\rho} \cdot A_{\rho}+A_{\mu}^{a} A_{\nu}^{b}\right]-\Lambda_{0}^{-4} \frac{\partial\left(A_{\mu}^{a} \Delta_{4}(A)\right)}{\partial A_{\nu}^{b}}+\cdots\right\},
$$

where the fields in the curly bracket are at the point $x$. The logarithm of the Jacobian is

$$
\operatorname{Tr} \ln \frac{\delta \mathcal{A}}{\delta A}=-S_{m}[\phi]+\mathcal{O}\left(\Lambda_{0}^{-2}\right)
$$

where $S_{m}[\phi]$ is the finite contribution for $\Lambda_{0} \rightarrow \infty$

$$
\begin{aligned}
S_{m}[\phi]= & \int d^{4} x\left\{7 c_{5} \Lambda_{0}^{2} A_{\nu} \cdot A_{\nu}+7 c_{0} A_{\nu} \cdot \partial^{2} A_{\nu}-7 c_{1}\left(\partial_{\nu} A_{\nu}\right)^{2}+15 c_{2}\left(\partial_{\nu} A_{\rho}\right) \cdot A_{\rho} \wedge A_{\nu}\right. \\
& \left.+4 c_{3}\left(A_{\rho} \wedge A_{\nu}\right) \cdot\left(A_{\nu} \wedge A_{\rho}\right)+\left(4 c_{4}+\frac{5}{2} c_{5}^{2}\right)\left(A_{\nu} \cdot A_{\nu}\right)^{2}\right\}
\end{aligned}
$$

Therefore, although (3) formally is trivial for $\Lambda_{0} \rightarrow \infty$ the logarithm of the Jacobian does not vanish in this limit but contains six relevant terms. Among these parameters one has a gauge mass term proportional to the UV scale.

\section{Effective action and ST identities}

We now consider the effective action $\Gamma[\phi, \gamma]$ obtained by adding to the UV action the source term expressed in terms of the effective fields $\phi$

$$
(j, \phi)=\int d^{4} x\left\{j_{\mu}(x) \cdot A_{\mu}^{\mathrm{R}}(x)+\bar{\chi}(x) \cdot c^{\mathrm{R}}(x)+\bar{c}^{\mathrm{R}}(x) \cdot \chi(x)\right\} .
$$

We introduce the renormalized fields and BRS sources $\phi^{\mathrm{R}}, \gamma^{\mathrm{R}}$ related to $\phi, \gamma$ by normalization constants

$$
A_{\mu}=\sqrt{Z_{A}} A_{\mu}^{\mathrm{R}}, \quad c=\sqrt{Z_{c}} c^{\mathrm{R}}, \quad W_{\mu}=\sqrt{Z_{c}} W_{\mu}^{\mathrm{R}}, \quad v=\sqrt{Z_{v}} v^{\mathrm{R}} .
$$




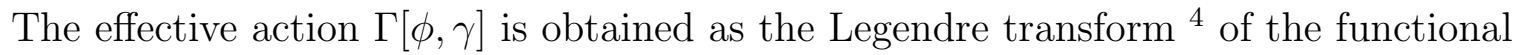

$$
Z[j, \gamma]=\int D \Phi \exp \left\{-S_{\mathrm{BRS}}\left[\Phi, \gamma, g_{\Lambda_{0}}\right]-S_{\text {cutoff }}[\Phi]+(j, \phi)\right\} .
$$

By making the field transformation (3) and using the Jacobian (9) we can write

$$
Z[j, \gamma]=\int D \phi e^{-S_{\Lambda_{0}}[\phi, \gamma]+(j, \phi)},
$$

where $S_{\Lambda_{0}}[\phi, \gamma]$ is the UV action in terms of the effective fields

$$
S_{\Lambda_{0}}[\phi, \gamma]=S_{\mathrm{BRS}}\left[\phi, \gamma, g_{\Lambda_{0}}\right]+S_{m}[\phi]+S_{\text {cutoff }}[\phi]+\cdots
$$

where the dots represent irrelevant contributions proportional to powers of $\Lambda_{0}^{-2}$. The relevant part $S_{\Lambda_{0}}[\phi, \gamma]$ depends on nine parameters: the six constants $c_{i}$ in the field transformation (3), the UV coupling $g_{\Lambda_{0}}$ and the three normalization constants of the field and BRS sources (12). Notice that $c_{0}$ has not to be considered as an independent parameter since it can be absorbed in $Z_{A}$.

While the UV action $S_{\mathrm{BRS}}\left[\Phi, \gamma, g_{\Lambda_{0}}\right]$ and the measure $D \Phi$ are both invariant under the BRS transformation (5), the UV action $S_{\Lambda_{0}}[\phi, \gamma]$ in (15) and the measure $D \phi$ are not invariant under the BRS transformation (6). This is due to the fact that the logarithm of the Jacobian from $\Phi$ to $\phi$ has been included into $S_{\Lambda_{0}}[\phi, \gamma]$.

We come now to the question whether the effective action obtained from (14) does satisfy the ST identities in the limit $\Lambda_{0} \rightarrow \infty$. As mentioned at the beginning the requirement that $\Gamma[\phi, \gamma]$ satisfy the ST identities fixes (perturbatively) the six parameters $c_{i}$ of the field transformation (3). Moreover all polynomials in (3) with dimension higher than four can be neglected. These properties are consequences of the well known results on renormalizability and ST identities in gauge theories (see for instance [5] and therein references).

The mentioned results have been recently rederived [5], [6] within the framework of exact RG in which one explicitly deals with a momentum cutoff and a UV action given by the relevant part of $S_{\Lambda_{0}}[\phi, \gamma]$. One shows perturbatively that, by properly fixing the nine relevant parameters in $\Gamma[\phi, \gamma]$, the effective action remains finite for $\Lambda_{0} \rightarrow \infty$ (renormalizability) and satisfies ST identities. Among these parameters one has the physical coupling $g$ at a subtraction point $\mu \neq 0$.

By using the RG equations one can then compute in terms of $g$ and $\mu / \Lambda_{0}$ the nine relevant parameter in the UV action $S_{\Lambda_{0}}[\phi, \gamma]$, obviously the same number of relevant parameters in $\Gamma[\phi, \gamma]$. In particular one computes the six UV constants of the field transformation (3). For the explicit values at one loop see [6]. At one loop one finds that all $c_{i}$ with $i \geq 1$ are finite for $\Lambda_{0} \rightarrow \infty$. Recall that $c_{0}$ contributes to the normalization of the gauge field.

\section{Summary and comparison with LGT}

\footnotetext{
${ }^{4}$ In the path integral the source $j$ are coupled to renormalized field $\phi^{\mathrm{R}}$ as in (11). However, for simplicity of notation, we denote by $\phi$ the fields in the effective action, i.e. the "classical" fields conjugate to the source $j$ in the Legendre transform of $Z[j, \gamma]$. Similarly, in the effective action we denote by $\gamma$ the BRS source.
} 
By following the example of LGT we have shown that it is possible in the continuum to formulate a gauge theory in which both the effective action satisfies ST identities and the "bare" action at the UV cutoff is BRS invariant. The key point is the assumption that the field in the UV action are different from the fields in the effective action. The main difference between the continuous and the lattice formulation is in the structure of the field transformation. It is useful to summarize here these differences and the consequences.

Continuous case. The field transformation (3) is constructed in such a way to satisfy the Lorentz covariant structure of the field and, as a consequence, one has manifest Lorentz invariance in the UV action $S_{\Lambda_{0}}[\phi, \gamma]$. The field transformation involves infinite number of parameters but one needs to fix only the six coefficients of the field polynomials (4) of dimension two and four which give rise to relevant contributions to $S_{\Lambda_{0}}[\phi, \gamma]$. Polynomials in (3) of higher dimensions gives irrelevant contributions to $S_{\Lambda_{0}}[\phi, \gamma]$. The coefficients $c_{i}$ are fixed by the gauge symmetry, i.e. by properly fixing these coefficients one has that the effective action satisfies the ST identities.

Lattice gauge theory. The field transformation is given by the exponential mapping $U_{\mu}(x)=$ $\exp \left\{g_{\text {lat }} a A_{\mu}(x)\right\}$. By using the group property one can easily prove that the lattice effective action satisfies ST identities [8]. However, the transformation has manifest cubic covariance instead of the Lorentz one. From the logarithm of the Jacobian one finds that the UV action, written in terms of the gauge fields $A_{\mu}(x)$, contains relevant terms which

are non-scalar under Lorentz transformations. It contains also a gauge field mass term and infinite irrelevant couplings. While in the continuous case all irrelevant contributions in the UV action can be neglected, in the LGT case they are essential to cancel the various Lorentz non-scalar contributions.

We have benefited greatly from discussions with Giuseppe Burgio, Marco D'Attanasio, Claudio Destri and Enrico Onofri.

\section{References}

[1] C. Becchi, A. Rouet and R. Stora, Phys. Lett. 52B (1974) 344.

[2] K.G. Wilson, Phys. Rev. B 4 (1971) 3174, 3184; K.G. Wilson and J.G. Kogut, Phys. Rep. 12 (1974) 75.

[3] J. Polchinski, Nucl. Phys. B231 (1984) 269.

[4] G. Gallavotti, Rev. Mod. Phys. 57 (1985) 471.

[5] C. Becchi, On the construction of renormalized quantum field theory using renormalization group techniques, in Elementary particles, Field theory and Statistical mechanics, Eds. M. Bonini, G. Marchesini and E. Onofri, Parma University 1993.

[6] M. Bonini, M. D’Attanasio and G. Marchesini, Phys. Lett. 346B (1995) 87;Nucl. Phys. B437 (1995) 163. 
[7] K.G. Wilson, Phys. Rev. D 10 (1974) 2445. See also Lattice gauge theories and Monte Carlo simulations, ed C. Rebbi, World Scientific 1983 and, for a recent presentation, I. Montvay and G. Munster, Quantum fields on a lattice, Cambridge University press. 1994.

[8] H. Kawai, R. Nakayama and K. Seo, Nucl. Phys. B189 (1981) 49; T. Reisz, Nucl. Phys. B318 (1989) 417; S. Caracciolo, P. Menotti and A. Pelissetto, Nucl. Phys. B375 (1992) 195. 